RESEARCH ARTICLE SUMMARY

AVIAN GENOMICS

\section{Core and region-enriched networks of behaviorally regulated genes and the singing genome}

Osceola Whitney, ${ }^{* \dagger}$ Andreas R. Pfenning, ${ }^{*+}$ Jason T. Howard, Charles A. Blatti, Fang Liu, James M. Ward, Rui Wang, Jean-Nicolas Audet, Manolis Kellis, Sayan Mukherjee, Saurabh Sinha, Alexander J. Hartemink, Anne E. West,* Erich D. Jarvis*
INTRODUCTION: Brain activity drives both behavior and regulated gene expression in neurons. Although past studies have identified activity-induced signaling and gene regulation cascades in cultured neurons, much less is known about how activity-dependent transcriptional networks are affected by the variations in cell-type composition, network interconnections, and firing patterns that comprise behaviorally active brain circuits in vivo.

RATIONALE: We tested the hypothesis that behaviorally regulated gene expression is anatomically and temporally diverse and that the key determinants of this diversity are networks of transcription factors, their genomic binding sites, and epigenetic chromatin states. We analyzed genome-wide, singingregulated gene expression across time in the four major forebrain regions of the song control system in songbirds, a model of speech production in humans. We then performed a transcription factor motif analysis to identify gene regulatory networks enriched in each song nucleus and measured acetylation of histone 3 at lysine 27 (H3K27ac) to identify chromatin regions that were transcriptionally active in the genomes of song nuclei before and after singing.

RESULTS: We found that singing was associated with differential regulation of about $10 \%$ of all genes in the avian genome that came in several waves across time. Less than $1 \%$ of these genes were comparably regulated in all song nuclei tested, and these comprised a core set dominated by immediate-early gene (IEG) transcription factors. By contrast, the vast majority of singing-regulated genes were regulated in only one or a subset of song nuclei, such that each song nucleus had its own dominant subset of genes regulated with defined temporal profiles, controlling a variety of functions. The promoters of many of the singing-regulated genes contained binding motifs for known early-activated transcription factors (EATFs) that become active in response to neural firing, some of which were expressed differentially between song nuclei at baseline. One EATF, calciumresponse factor $(C a R F)$, was tested with RNA interference knockdown in cultured neurons

\section{ON OUR WEB SITE}

Read the full article at http://dx.doi .org/10.1126/ science. 1256780 nucleus-specific singing-regulated genes did not show increased chromatin regulatory element activity after singing but rather already had primed region-specific regulatory activity before singing began.

CONCLUSIONS: We propose a dual mechanism for the diversity of behaviorally regulated genes across different brain regions in vivo (see the figure). First, the neural activity associated with singing activates EATFs, and some TFs differentially expressed in brain regions at baseline, to trigger region-specific expression of their target genes. Second, brain region-specific enhancers near activity-regulated genes are waiting in an epigenetically primed state, ready to modulate transcription of general and song nucleusspecific genes at a moment's notice when the neurons fire. The combination of these two mechanisms underlies a great diversity of behaviorally regulated gene expression, permitting each nucleus to perform its particular function in this complex behavior.

The list of author affiliations is available in the full article online. *Corresponding author. E-mail: owhitney@gmail.com (O.W.); apfenning@csail.mit.edu (A.R.P.); west@neuro.duke.edu (A.E.W.); jarvis@neuro.duke.edu (E.D.J.)

Cite this article as 0 . Whitney et al.,Science 346, 1256780 (2014). DOI: $10.1126 /$ science.1256780 line. More strikingly, we

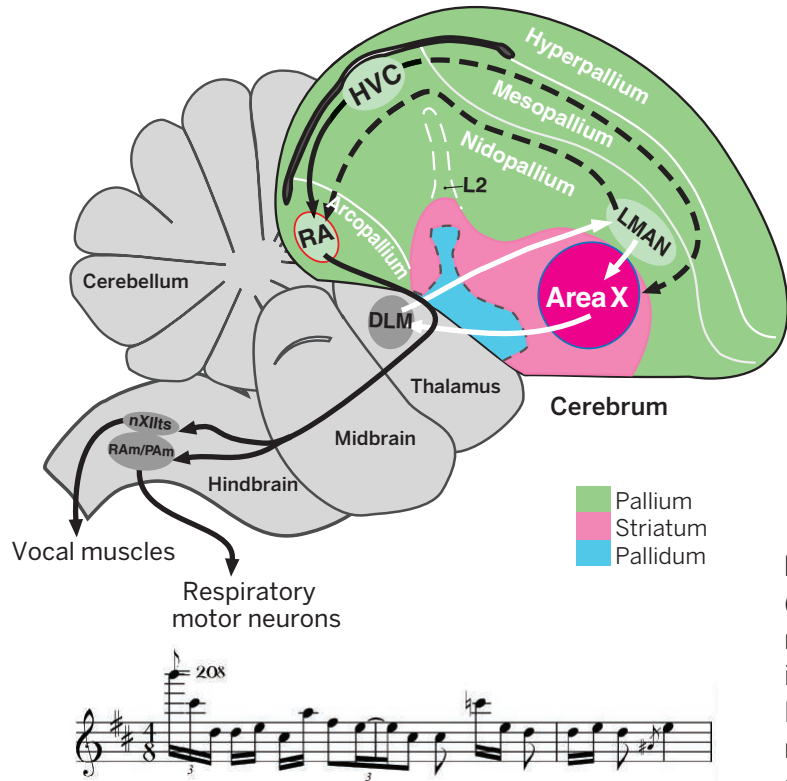

Singing-induced genes
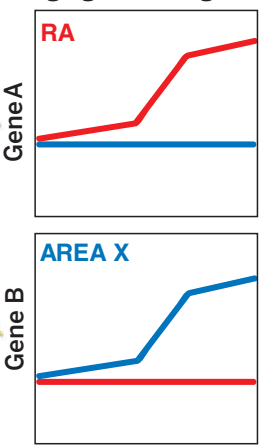

Time
H3K27ac primed
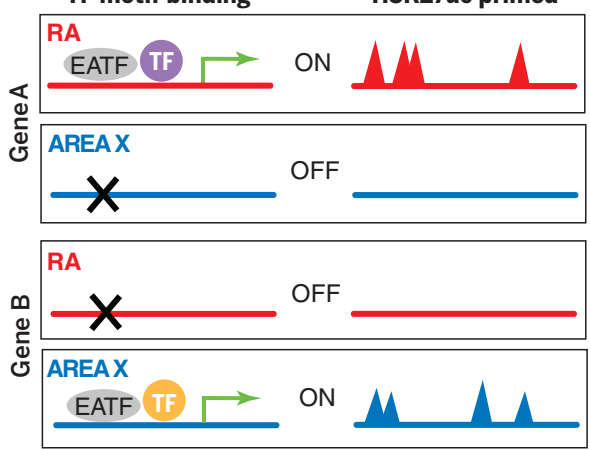

Brain region-specific genomic activation
Dual mechanism model for behaviorally regulated gene expression diversity. (Left) Song brain circuit and zebra finch song motif (transcribed using https:// my.scorecloud.com). (Middle) Song nucleus-specific (RA, red; Area X, blue) singing-regulated genes (gene A and gene B) in response to neural firing (yellow). (Right) Region-general EATF and region-specific TF only bind to genomic DNA (lines) with region-specific acetylated histone 3 (H3K27ac peaks) and then transcribe their mRNAs (green arrow). 


\section{Core and region-enriched networks of behaviorally regulated genes and the singing genome}

\author{
Osceola Whitney, ${ }^{1 *} \dagger$ Andreas R. Pfenning, ${ }^{1,2 *}+$ Jason T. Howard, ${ }^{1}$ \\ Charles A Blatti, ${ }^{3}$ Fang Liu, ${ }^{4}$ James M. Ward, ${ }^{1}$ Rui Wang, Jean-Nicolas Audet, ${ }^{5}$ \\ Manolis Kellis, ${ }^{2}$ Sayan Mukherjee, ${ }^{6}$ Saurabh Sinha, ${ }^{3}$ Alexander J. Hartemink, ${ }^{7}$ \\ Anne E. West, ${ }^{4 *}$ Erich D. Jarvis ${ }^{1 *}$
}

\begin{abstract}
Songbirds represent an important model organism for elucidating molecular mechanisms that link genes with complex behaviors, in part because they have discrete vocal learning circuits that have parallels with those that mediate human speech. We found that $\sim 10 \%$ of the genes in the avian genome were regulated by singing, and we found a striking regional diversity of both basal and singing-induced programs in the four key song nuclei of the zebra finch, a vocal learning songbird. The region-enriched patterns were a result of distinct combinations of region-enriched transcription factors (TFs), their binding motifs, and presinging acetylation of histone 3 at lysine 27 (H3K27ac) enhancer activity in the regulatory regions of the associated genes. RNA interference manipulations validated the role of the calcium-response transcription factor (CaRF) in regulating genes preferentially expressed in specific song nuclei in response to singing. Thus, differential combinatorial binding of a small group of activity-regulated TFs and predefined epigenetic enhancer activity influences the anatomical diversity of behaviorally regulated gene networks.
\end{abstract}

S ongbirds offer an important in vivo model system for studying transcriptional programs regulated during behavior. This system consists of interconnected brain nuclei that control production of a learned vocal behavior (singing) with parallels to human speech $(1,2)$. Four key song nuclei are embedded within three regionally distinct telencephalic brain cell populations: HVC (letter based name), LMAN (lateral magnocellular nucleus of the nidopallium), RA (robust nucleus of the arcopallium), and Area $\mathrm{X}$ in the striatum. (Fig. 1A) (3-6). These nuclei are connected in a vocal motor pathway (HVC to RA) and a vocal learning pathway (LMAN and Area X) (7-13). Human functional analogs to these avian brain regions are in the cortex (pallium) and basal ganglia (striatum) $(2,6,14,15)$. This includes song (avian) and speech (human) brain regions that have convergence of differentially expressed genes (15), which suggests that the behavioral and neuroanatomical similarities for the production of

\footnotetext{
Department of Neurobiology, Howard Hughes Medical Institute, and Duke University Medical Center, Durham, NC 27710, USA. ${ }^{2}$ Computer Science and Artificial Intelligence Laboratory and the Broad Institute of MIT and Harvard, Massachusetts Institute of Technology, Cambridge, MA 02139, USA. ${ }^{3}$ Department of Computer Science, University of Illinois, Urbana-Champaign, IL, USA. ${ }^{4}$ Department of Neurobiology, Duke University Medical Center, Durham, NC 27710, USA. ${ }^{5}$ Department of Biology, McGill University, Montreal, Quebec H3A 1B1, Canada. ${ }^{6}$ Department of Statistics, Duke University, Durham, NC, USA. ${ }^{7}$ Department of Computer Science, Duke University, Durham, NC 27708-0129, USA. *Corresponding author. E-mail: owhitney@gmail.com (O.W.); apfenning@csail.mit.edu (A.R.P.); west@neuro.duke.edu (A.E.W.); jarvis@neuro.duke.edu (E.D.J.) †These authors contributed equally to this work.
}

learned vocalizations are accompanied by similarities in molecular and genetic mechanisms, such as with FoxP2 (16).

The neural activity within song nuclei that underlies singing was initially shown to drive induction of two immediate early genes (IEGs), the transcription factors EGRI and FOS (17-19). Their levels of expression correlate with the amount of singing in a motor-driven and socialcontext-dependent manner (20-23). Subsequent studies identified an additional 33 genes regulated within song nuclei by singing (24). The identified gene products have a wide range of cellular and biological process functions (24), including from neurogenesis $(25,26)$ to speech $(27,28)$. The genes were also found to cluster in a few anatomical and short temporal patterns of expression, although this was determined manually. As a result, we hypothesized that in vivo behaviorally induced gene expression may consist of anatomically and temporally diverse gene expression programs that can be regulated by networks of combinatorial transcription factor complexes or epigenetic chromatin differences (24). Two reports $(29,30)$ using our oligonucleotide microarrays found many more genes -800 to 2000 gene transcripts-regulated in the song nucleus Area $\mathrm{X}$ as a result of singing but could not test this hypothesis because the data were from only one song nucleus and/or one time point.

To test this hypothesis, we profiled baseline and singing-regulated gene expression across time in the four key song nuclei using our songbird gene expression microarray, which we annotated based on recently sequenced avian genomes $(15,31)$ and the human genome. Combined with genomic transcription factor motif analyses and chromatin immunoprecipitation sequencing (ChIP-seq) detection of active chromatin, we found predominantly diverse networks of simultaneously activated cascades of behaviorally regulated genes across brain regions, which can be explained in part by a combination of transcription factor complexes and epigenetic regulatory activity in the genome.

\section{Results}

We analyzed singing-regulated gene expression at a genomic-scale in HVC, LMAN, RA, and Area $\mathrm{X}$ of the zebra finch (Fig. 1 and fig. S1). To do so, we recorded moment-to-moment singing behavior of all animals over a 7-hour time course, laser microdissected individual song nuclei from multiple birds at each time point, amplified their mRNA, hybridized the resulting cDNA to our custom-designed $44 \mathrm{~K}$ oligonucleotide microarrays (table S1), and developed a computational approach that yielded a true positive rate $>87 \%$, as verified by in situ hybridization and reverse transcription polymerase chain reaction [fig. S2; tables S2 and S3; supplementary materials sections 1 to 7 (SM1 to SM7)]. This analysis detected 24,498 expressed transcripts among the four song nuclei in silent and/or singing animals (table S4), of which 18,478 (75\%) mapped to 9059 Ensembl v60 annotated genes of the zebra finch genome, indicating that at least $50 \%$ of the transcribed genome is expressed in the song-control circuit of an adult animal during awake behaving hours.

\section{Distinct baseline gene expression profiles define the song circuit}

Using a linear model that we developed to identify differentially expressed transcripts in each brain region and combinations thereof (SM6), we found that of the 24,498 transcripts, $\sim 5167$ $[21 \%$, representing 3168 genes or $\sim 17 \%$ of the genes in the avian genome (29)] were differentially expressed among song nuclei at baseline in silent animals (i.e., before singing began). These 5167 transcripts were organized hierarchically into at least five major region-specific clusters (Fig. 2A and table S5) with different functional enrichments (tables S6 and S7). A striatal song nucleus (Area $\mathrm{X}$ ) cluster was enriched with noncoding RNAs, G protein-coupled receptors, and synaptic transmission proteins (Fig. 2A, turquoise cluster, and table S6). Corticallike song nuclei (HVC, LMAN, and RA) were enriched for cell-to-cell signaling membraneassociated, axonal connectivity, and postsynaptic density (PSD) proteins (Fig. 2A, blue cluster, and table S6). The nidopallium song nuclei (HVC and LMAN) were further enriched for another group of cell-cell communication and neural connectivity, membrane-associated proteins (Fig. 2A, yellow cluster, and table S6). The arcopallium song nucleus RA was enriched for another set of neural connectivity proteins and for proteins involved in epilepsy and Alzheimer's (Fig. 2A, green cluster, and table S6). RA was the only 


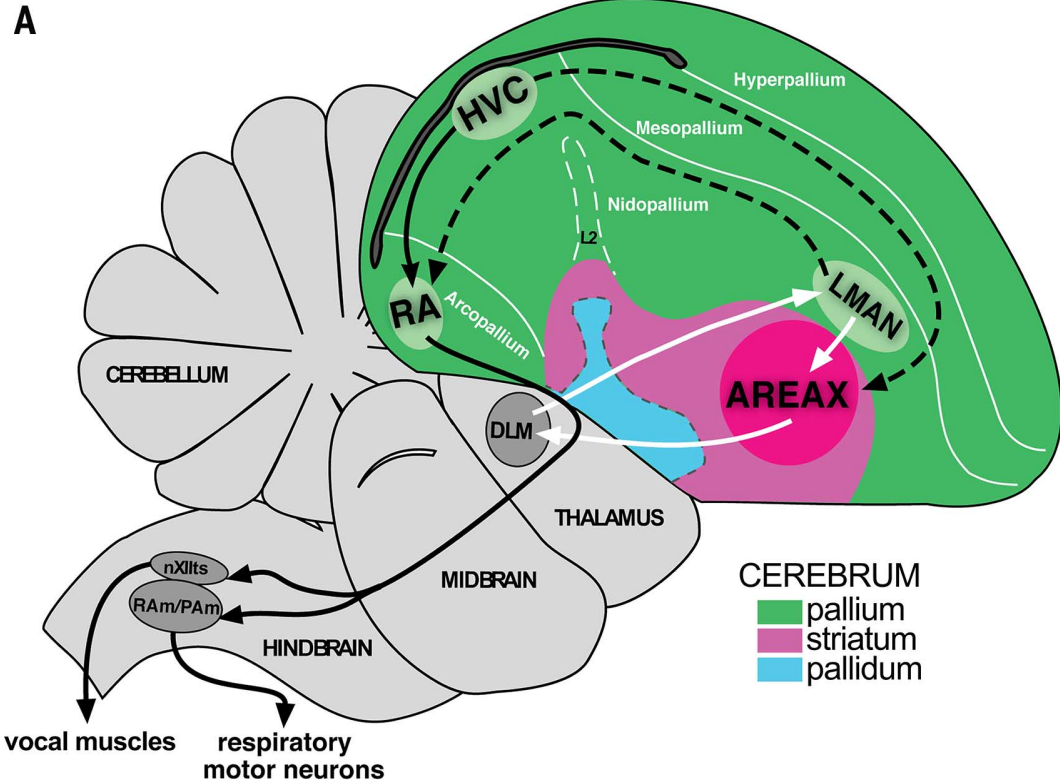

B
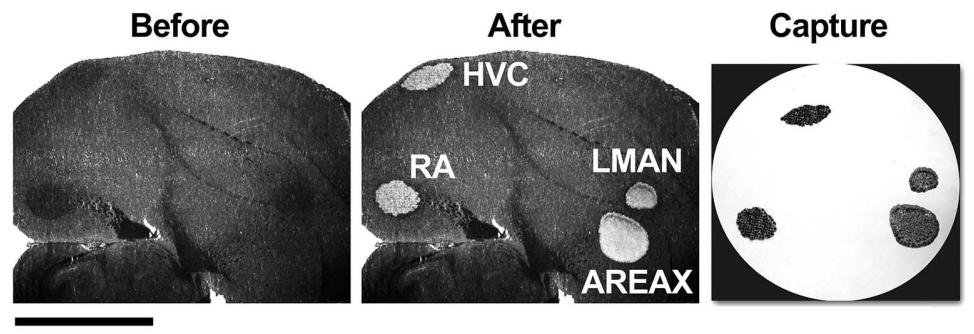

Fig. 1. Song system and laser microdissection. (A) Sagittal schematic of the zebra finch brain showing positions and some connections of song nuclei. Pallial, striatal, and pallidal regions are distinguished by colors. Black arrows, posterior vocal pathway involved in song production; white arrows, anterior vocal pathway involved in song learning and modulation; dashed arrows, connections between the two pathways. (B) Song nuclei were laser-capture microdissected from males that were either silent or continuously singing for 0.5 hours and 1 hour, and for each hour thereafter up to 7 hours, resulting in more than 200 total microarrays. Shown are images of $10-\mu \mathrm{m}$ tissue sections before and after laser capture microdissection at 10X magnification. (Before) Following dehydration, song nuclei fiber density appears darker than surrounding tissue. (After) Song nuclei regions are selectively cut out using an infrared laser. (Capture) The cut song nuclei transferred to the cap by the LCM system. For microarray analysis, each of the four song nuclei from each animal was captured separately to individual LCM caps. Dorsal is up; anterior is right. Scale bar, $2 \mathrm{~mm}$.

pallial brain region that had a large cluster of genes with a lower level of expression, which was enriched for PSD proteins different from the cortical enrichment (Fig. 2A, brown cluster, and table S6), and LMAN was the only song nucleus that did not have a large enrichment of genes of its own.

In situ hybridizations of example genes (e.g., some dopamine and glutamate receptors) revealed that most of the song nuclei expression patterns were consistent with the brain subdivisions to which they belonged (Fig. 3, A to C, and table S2) (32-34). However, as seen previously $(33,35,36)$, some of the song nuclei had highly differential expression from their surrounding brain divisions (i.e., FMNL1, DGKI, and GPSM1 in Fig. 3, A to C). The most song-nucleus-specific gene was FAM4OB (also called STRIP2), a phosphatase that was restricted to cortical-like song nuclei and the primary cortical sensory populations (like auditory area L2 in Fig. 3A).

A dendrogram analysis separated the cortical song nuclei from the striatal and showed a stronger relationship between HVC and LMAN of the nidopallium (Figs. 2B and 1A), consistent with the recently revised understanding of avian brain organization and homologies with mammals $(5,6,37)$. These findings show that even before singing starts, the song-learning nuclei have thousands of differentially expressed genes that define specific molecular functions for each [see (15) for characterization of the specializations in song nuclei].

\section{Singing activates both a core and regionally diverse patterns of genes}

Of the 24,498 transcripts, we found an estimated $2740(\sim 11 \%)$ that were singing-regulated, up or down in time, in one or more song nuclei (Fig. 4, $\mathrm{A}$ and $\mathrm{B}$, and table S8). These transcripts mapped to 1833 genes, indicating a conservative estimate of $\sim 10 \%$ of the transcribed avian genome that is regulated by singing behavior. Area $\mathrm{X}$ had the most regulated transcripts (1162), followed by HVC (772), RA (702), and LMAN (635) (Fig. 4B) (the sum is higher than 2740 because of transcripts expressed in more than one song nucleus). A small number of genes (82) had singingregulated splice variant differences (table S9), consistent with splice variant differences at baseline among song nuclei for glutamate receptor subunits (33), which can regulate activity-dependent genes in the brain. The vast majority (96\%) of the 2740 singing-regulated transcripts were enriched in only one or two song nuclei, and a core set of only about 97 transcripts was regulated in at least three or four $(<1.0 \%)$ song nuclei; of the latter, only 20 genes were equally regulated in all four song nuclei (Fig. 4, A and B, and table S8, green and yellow).

The core set of 97 transcripts was enriched for known IEGs (38), including membrane depolarization-regulated $\left(\mathrm{Ca}^{2+}\right.$ responsive) genes identified in cultured hippocampal (39) and cortical neurons (40) and genes induced in the auditory pathway by hearing song (41) (tables S10A and S7). In contrast, the brain region-specific singing-regulated genes had very little overlap with classic IEGs or a list of cell cultured-defined depolarization-induced genes (table S1OA). Rather, the striatal Area X singing-regulated genes were enriched for cytoskeletal neural connectivity and neural migration functions, and RA was enriched for mitogen-activated protein kinase pathway transcripts, which control gene expression, differentiation, and cell survival. This suggests that our in vivo analyses are useful for finding region-specific or stimulus-specific genes that may be relevant for the underlying singing behavior.

Similar to the baseline expression, in situ hybridizations revealed that song nuclei expression patterns were consistent with the brain subdivisions to which they belong (Fig. 3, A to C, and table S3), except that the surrounding brain areas in some birds tended to have lower expression, presumably because they sang without much other movement behavior to cause movementinduced gene expression in the surrounding regions (42). We also noted that even among the core early-response genes induced in all song nuclei, expression levels at baseline differed among song nuclei (Fig. 3D). This suggests that there is even greater diversity among the song nuclei singing-regulated genes than simply presence or absence of regulation.

Analysis of the behaviorally regulated gene expression across time, using unsupervised hierarchical clustering (SM8), revealed up to 20 temporal profiles (clusters) among the four song nuclei, including transient or sustained, increased or decreased, early ( 0.5 to 2 hours) or late (3 to 7 hours), or two peaks of expression (fig. S3, A to D, and table S8). These 20 clusters can be further grouped into four superclusters 


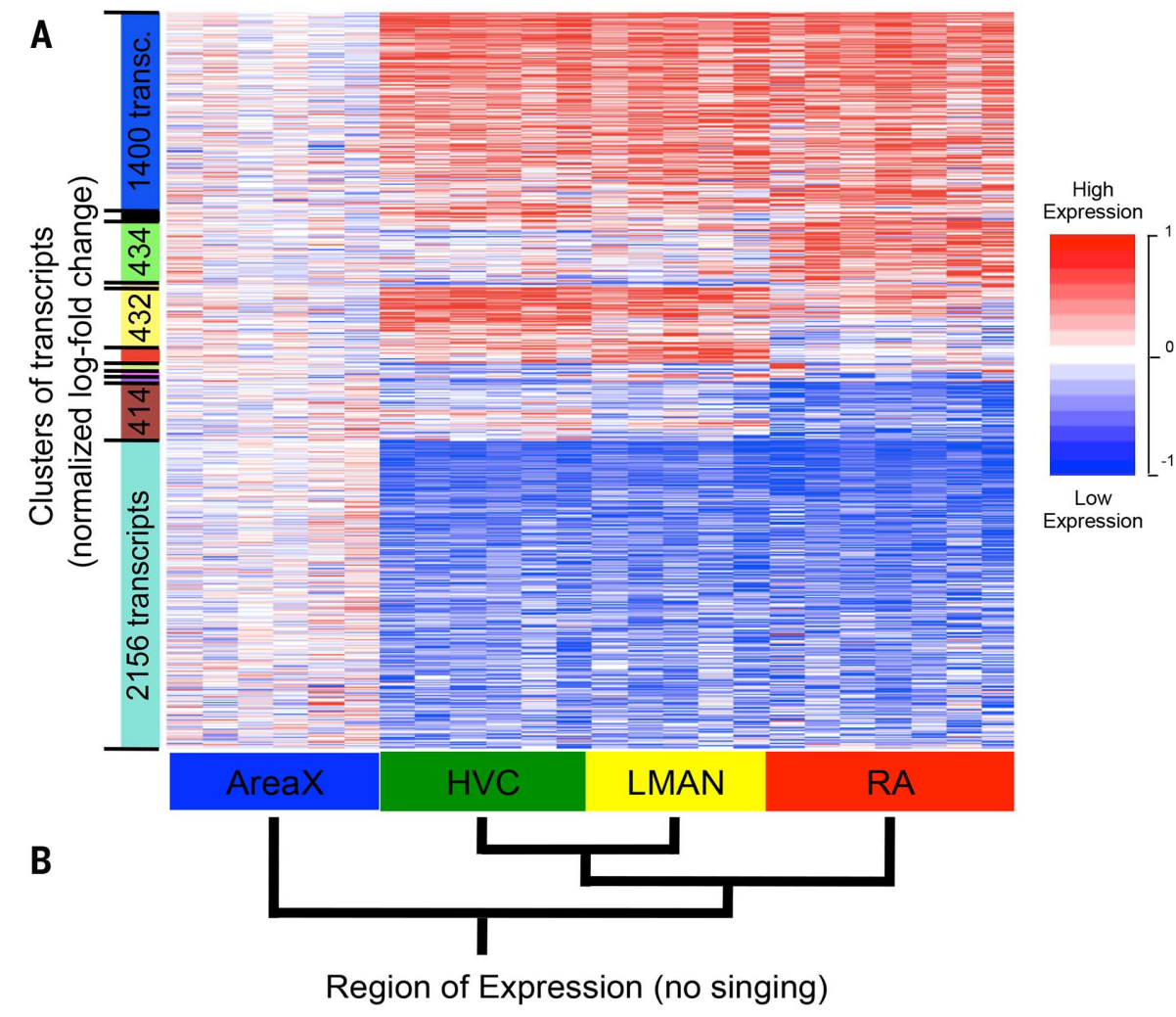

Fig. 2. Region-enriched gene expression at baseline. (A) A heat map of hierarchically clustered expression profiles of 5167 transcripts (rows) that are differentially expressed across regions at baseline (FDR $q<0.1$; see fig. S11 for FDR $q<0.2$ ) in silent birds (red, increases; blue, decreases; white, no change) relative to mean Area $X$ expression (numbers of transcripts not shown for small clusters). Each transcript is normalized to the average value of expression in Area X. Each column is an animal replicate. Detailed results are in table S4. (B) Average linkage hierarchical tree, generated from mean expression in each brain region, representing the molecular expression relationships between regions.

of temporal profiles: (i) transient early increases, (ii) late-response increases, (iii) transient early decreases, and (iv) late-response decreases (Fig. 5, A to D). Only three of the temporal clusters had relatively comparable representations of genes in all brain regions, all belonging to transient early-increase clusters, including the IEG 0.5 to 1 hour cluster (Fig. 5A; fig. S3, tan cluster; and table S11), which contained a significant proportion $(16 \%)$ of the core set of 97 transcripts $(P<$ $1 \times 10^{-5}$, hypergeometric test). For the remaining supertemporal profiles, each song nucleus had a region-enriched set of genes, except the lateresponse increasing pattern in LMAN (Fig. 5, fig. S3E, and table S11).

Functional enrichment analyses showed that the activity-regulated gene expression sets from previous cell culture experiments (table S7) were highly enriched in the early transient IEG temporal cluster expressed in all song nuclei (table S10B). All of the late-increase singing-regulated clusters (Fig. 5B) also had detectable functional enrichments of genes, with Area X+HVC enriched in calcium ion binding and phosphatase proteins (blue temporal cluster); Area X late-increase genes were additionally enriched in chromosome organization, biogenesis (green), activity-dependent expressed in most brain regions could regulate a diverse set of downstream genes, with little overlap among regions. We hypothesized that the differential transcriptional state at baseline, before cell stimulation with singing, affects region-enriched singing-regulated expression $(43,44)$. Three lines of evidence support this hypothesis. First, hypergeometric tests revealed significant overlap between subsets of transcripts from the baseline region-enriched clusters (Fig. $4 \mathrm{C}$, top gray box) with the singing-regulated region-enriched clusters (Fig. 4C, red lines and table S12) and with 10 of the 20 temporal clusters (Fig. 4C, blue and black lines between two gray boxes). If a gene was expressed at higher levels in a region relative to others at baseline before singing, it was also more likely to increase in that region during singing; the converse was not true for the decreasing sets of singingregulated genes.

Second, a genome-wide binding site analysis of motifs for transcription factors (SM11) $(45,46)$ revealed $\sim 100$ motifs enriched in regulatory regions (e.g., directly upstream of transcription start sites) of genes in the temporal behaviorally regulated clusters (tables $\mathrm{S} 13$ and S14 and Fig. 6, A and B), and these matched genomic locations were also found in mammalian genomes $(47,48)$. With these motifs, we performed an association analysis between the region-specific and temporal clusters of genes to generate song nuclei-specific transcription factor motif to gene cluster networks (Fig. 6C, simplified network; fig. S4, detailed network; and table S15, edge list) [statistical significance tested with Euclidean distance to randomly generated networks (SM11 and SM12)]. Consistent with the core IEG cluster findings, we found that binding sites for five early-activated transcription factors (EATFs) (MEF2, SRF, NFKB, CREB, and CaRF) that are constitutively expressed at baseline and activated in response to neural activity $(38,49,50)$ were significantly overrepresented in the singing-regulated cluster of IEGs expressed in most song nuclei (Fig. 6C and figs. S4 and S5A). In turn, the binding motifs of the singing-regulated $A P-1$ (bound by a FOS-JUN dimer) and EGRI IEG transcription factors were also enriched directly upstream of the transcription start sites of many genes in our avian IEG cluster (Fig. 6, A to C). EGRI can bind to its own promoter and downregulate itself (51), which is consistent with the transient increase and subsequent decrease of some transcripts in the IEG temporal cluster. Also overrepresented in the IEG cluster was the $A R N T$ motif, which also has the binding motif for the IEG NPAS4.

Third, consistent with our region-specific clusters, some transcription factors that were differentially expressed in a region or a combination of regions at baseline had binding motifs in genes that were differentially regulated in that region(s) at baseline or during singing. For example, variants of the NFE2L1 and MAF transcription factors that dimerize and bind to the TCF11 motif (52) were higher or lower in Area $\mathrm{X}$ relative to the pallial song nuclei at baseline (fig. S6), and 
A All Pallial Cluster

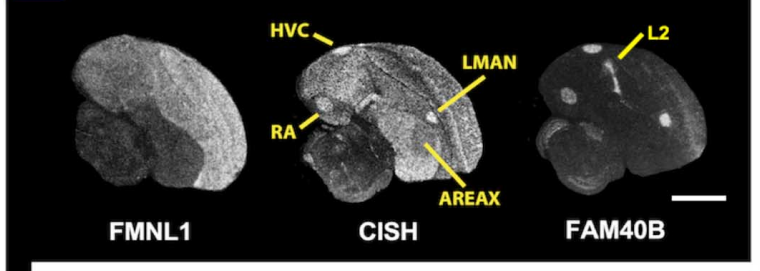

B Any Pallial Cluster

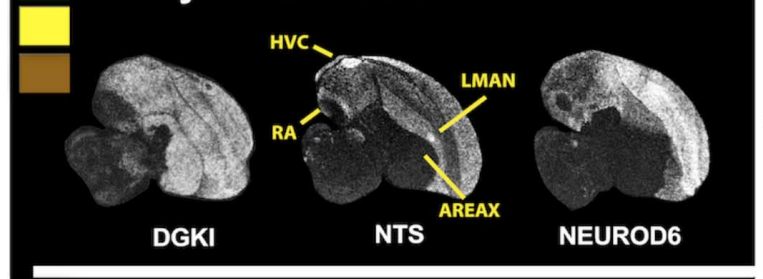

C Striatal Cluster
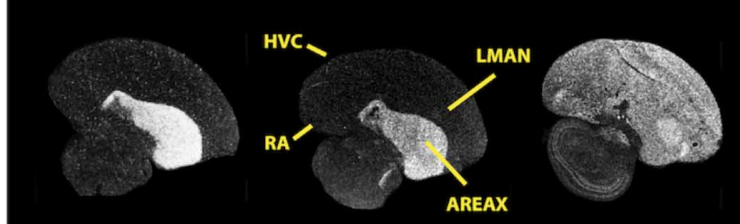

GPSM1
DLX6

RPH3DM1
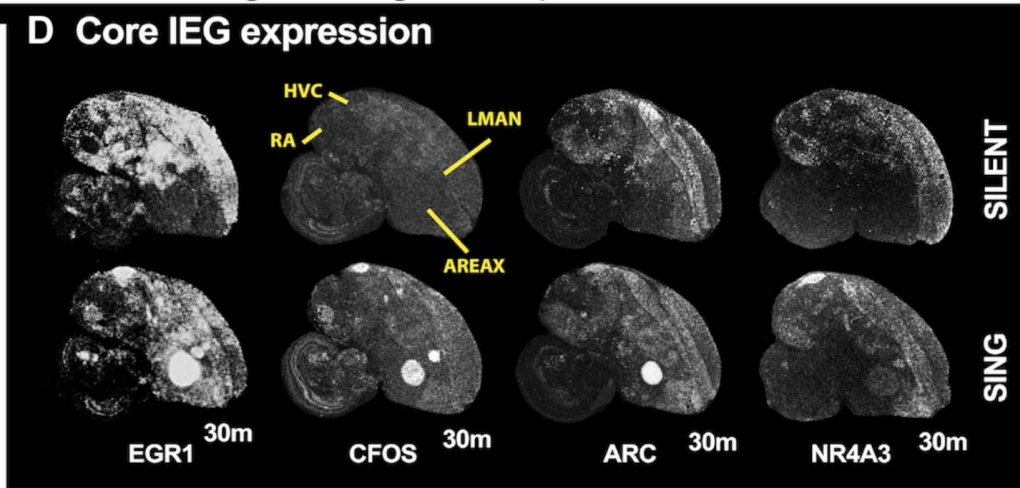

\section{E Region Specific}

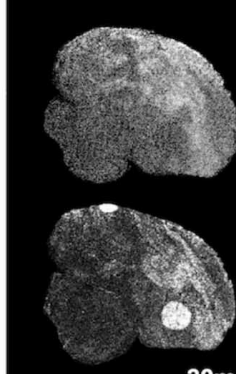

EGR2

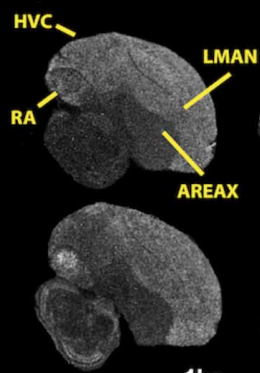

NFIL3

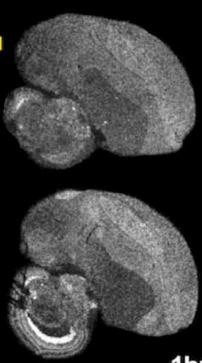

CSDC2

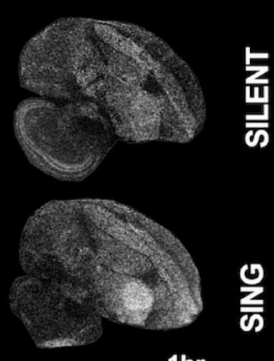

PTPN5

Fig. 3. In situ hybridizations of baseline and singing-regulated genes. (A) Genes higher in all pallial song nuclei (RA, HVC, and LMAN) relative to the striatal song nucleus (Area X) at baseline (Fig. 2A, blue clusters). (B) Genes differentially expressed just among the pallial song nuclei (green, yellow, and brown clusters) at baseline. (C) Genes higher in the striatal song nucleus relative to pallial song nuclei (turquoise cluster). (D) Core singing-regulated

genes regulated in three to four song nuclei detected by microarrays but detected in all four with diverse levels by in situ hybridization, most peaking at $30 \mathrm{~min}$. (E) Region-enriched singing-regulated genes in one or two song nuclei, with peaks of expression at later time points. Film autoradiograph images are inverted, showing white as labeled mRNA expression of the gene indicated below the image. Dorsal is up; anterior is right. Scale bar, $2 \mathrm{~mm}$.

the TCF11 binding motif was overrepresented in the slow-increase singing-regulated cluster of genes in Area X (Fig. 6C and figs. S4 and S5B). However, there were many other cases where EATFs and other transcription factors did not exhibit differential regional baseline expression but had binding motifs enriched in clusters of singing-regulated genes specific for a song nucleus. For example, the EATF transcription factors $S R F$ and $C a R F$, which are not differentially expressed at baseline (table S5), had strong motif associations to singing-regulated genes in Area X and HVC. The MZF1 and PRRX2 transcription factors had associations with different sets of genes in Area $\mathrm{X}$ and RA (Fig. 6C and figs. S4 and S5B). Thus, we experimentally tested whether one of these EATFs, $C a R F$, regulated the predicted region-specific genes (Fig. 7).

\section{$\mathrm{CaRF}$ is required for regulation of both core and regional expressed sets of genes}

We investigated the $\mathrm{Ca}^{2+}$ responsive transcription factor $C a R F$ because the network analyses implicated it in both the regulation of the $\mathrm{Ca}^{2+}$ responsive IEGs that are induced in most song nuclei and some that are regionally enriched in Area X and HVC (Fig. 4C and fig. S6). Because we lacked an established zebra finch neural cell culture method to test $C a R F$ function, we used RNA interference (RNAi) against CaRF in cultured mouse cortical neurons and hybridized labeled cDNA to mouse oligonucleotide microarrays representing many of the same genes on our zebra finch oligonucleotide microarray (SM4). We identified a set of genes that showed decreased or increased expression after $C a R F$ knockdown independent of membrane depolarization (Fig. 7A and table S16), and many of these function in calcium signaling pathways (fig. S7 and table S17) (53). This is consistent with the proposed role of $C a R F$ in regulating neuronal gene expression under basal neural activity $(48,54)$, as both a repressor and activator $(48)$. Importantly, as predicted by our promoter motif analyses in birds, the ranked list of $\mathrm{CaRF}$ regulated genes showed enrichment for singingregulated genes that had a nearby $C a R F$ binding site $(P=0.0014$, Wilcox test) (Fig. 7B). This enrichment was highest in the set of genes regulated in Area X and HVC (Fig. 7B), supporting our network result (Fig. 6C).

CaRF RNAi knockdown also caused genes that were normally up-regulated by membrane depolarization to be suppressed to normal baseline levels and, conversely, genes that were normally down-regulated by membrane depolarization to be up-regulated (Fig. $7 \mathrm{C}$ and table S18). This suggests that $C a R F$ is required to buffer activity of these gene promoters under basal conditions such that they can become stimulus-responsive upon membrane depolarization. Importantly, this same set of membrane depolarization- and $C a R F$-regulated genes significantly overlapped with those that had the $C a R F$ binding site in the singing-regulated genes of the IEG (tan) cluster. They also significantly overlapped with several other clusters that were specifically up-regulated in Area X and HVC (Fig. 7D, magenta and cyan clusters; table S19; and fig. S3E). Genes that showed decreased expression preferentially in RA, but also in other song nuclei (fig. S3, yellow), after 2 to 3 hours of singing (the same amount of time the cultured cells were depolarized) had even greater overlap (Fig. 7D, yellow)

Overall, the findings demonstrate a requirement of the $C a R F$ transcription factor for baseline and activity-dependent regulation of some of the very same genes for which we found $C a R F$ binding motifs that are regulated at baseline and by singing in a region-specific manner, respectively. The calcium signaling and calcium ion-binding genes tended to increase during song production and were affected in the $C a R F$ knockdown experiments, which is evidence of consistent $C a R F$ function across species. We next sought an explanation of how EATFs that are 
Fig. 4. Region-enriched gene expression in response to singing. (A) A four-way Venn diagram showing regional singingregulated distribution of 2740 transcripts (FDR $q<$ 0.2). (B) Heat map of all 2740 transcripts from the Venn diagram, hierarchically clustered independently in all four song nuclei, then sorted by increased or decreased expression, and level of significance from highest to lowest in the linear model. Each column (170 total) is an animal replicate within a time point, and white lines separate time points. Red, increases; blue, decreases; white, no change relative to 0-hour samples for each song nucleus. Each transcript is normalized so that the maximum increase relative to nonsinging birds in any region is the darkest shade of red for increasing transcripts, and the maximum decrease is the darkest shade of blue for decreasing transcripts. Boxes highlight significant behaviorally regulated enrichment for each region (FDR $q<0.2$ for that region). Figure S12 shows a more stringent heat map of regionenriched expression with a
A

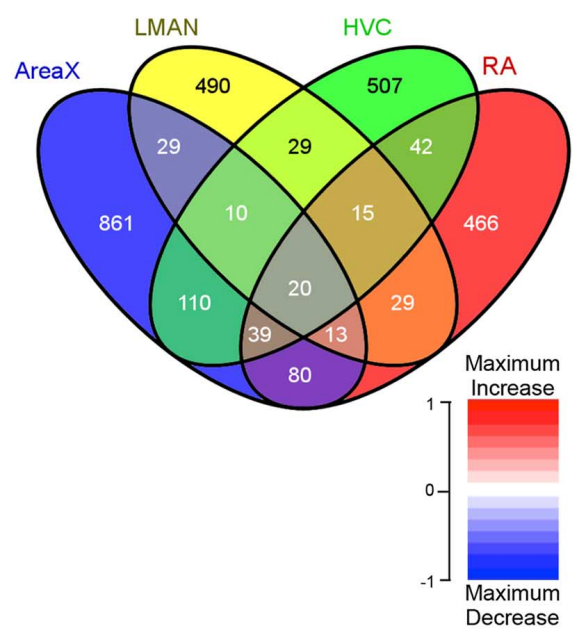

B

C

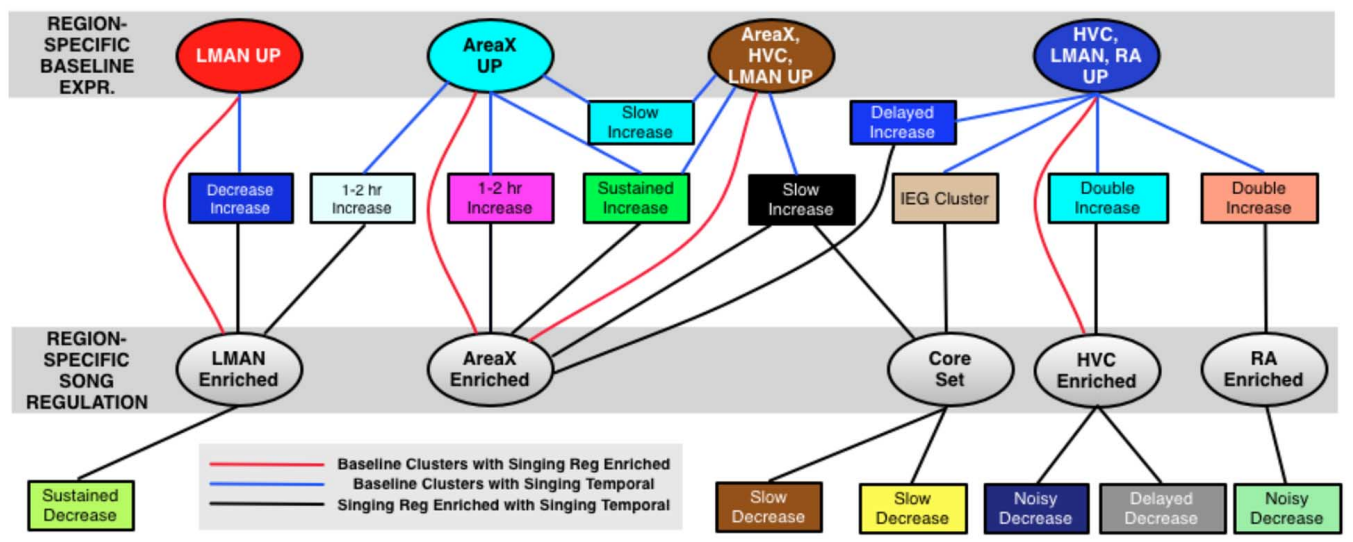

similar result. (C) Relationships among clusters of transcripts from the baseline region-enriched (top gray box, from Fig. 2A), singing temporal-enriched (rectangular nodes, from fig. S3, A to D), and singing region-enriched [bottom gray box, from (B)] patterns. Nodes are colored according to their cluster colors in the respective figures. Edges between two nodes correspond to significant overlap between two groups of transcripts $(P<0.001$, hypergeometric test). Nodes are sorted to optimize noncrossing of edges. Detailed results are in table S8.

not differentially expressed at baseline could regulate these genes in a region-specific manner.

\section{Epigenetic modifications predefine region specificity of gene regulation}

Although transcription factors are the ultimate regulators of gene expression, their ability to bind to sites in the genome is gated by chromatin structural changes. Chromatin regulation by acetylation of histone 3 at lysine 27 (H3K27ac) has been extensively studied and shown to be a strong indicator of active enhancers (55). We thus performed an experiment to identify active transcriptional regulatory regions in the genomes of individual dissected song nuclei (RA and Area $\mathrm{X}$, which showed the largest regional differences) before and after singing, as measured by a genome-wide histone ChIP-seq analysis of H3K27ac (SM14, SM15, and table S20). The active genomic regions can be searched as tracks in the University of California-Santa Cruz (UCSC) browser against the zebra finch genome (56). This analysis also required that we create a more stringent selection of regional, early, and late singing-responsive genes from the respective clusters in RA and Area X (Fig. 5 and fig. S3), using principal components analyses (fig. S8).

Out of 35,958 peaks, we found $30 \%(10,749)$ enriched in Area X and 21\% (7673) enriched in RA. Under basal conditions, genes with song nuclei-specific expression patterns had nearby genomic regions that were significantly more likely to be marked by H3K27ac in that brain region (Fig. 8A, blue and red, and table S21) ( $\sim 1300$ genes). Conversely, genes that were expressed similarly in RA and Area X did not show a significant regional bias in the distribution of this chromatin mark (Fig. 8A, gray, and table S21) ( $\sim 100$ genes examined). Interestingly, when we considered only the set of RA or Area X regionspecific genes that were also up-regulated by singing, we found that they were already associated with higher nearby H3K27ac in their preferred brain region before singing (Fig. 6, B, D, and E; fig. S9, A to E; and table S22). There was a strong positive correlation between differences in nearby H3K27ac at baseline and differences in singing-dependent up-regulation of these genes in RA and Area $\mathrm{X}(R=0.37, P=$ $1.6 \times 10^{-12}$; Pearson correlation). Conversely, late-response genes that were comparably induced by singing in both RA and Area X showed comparable H3K27ac under basal conditions (Fig. 8B, gray, and table S22). Furthermore, the early-response cluster of genes, which were expressed and induced comparably in both RA and Area X (e.g., FOS), also showed comparable H3K27ac in both brain regions at baseline (Fig. $8 \mathrm{C}$ and figs. S9A and S10A). Notably, we did not find any significant difference [e.g., 0 significant peaks; false discovery rate (FDR) threshold $<0.01]$ in H3K27ac peaks within either song nucleus when we compared ChIP-seq profiles obtained before and after singing (fig. S10A). We detected a weak signal for increased H3K27ac peaks in the Area X down-regulated genes (fig. S10B).

These data suggest that the regional differences in chromatin activity present before singing 
A

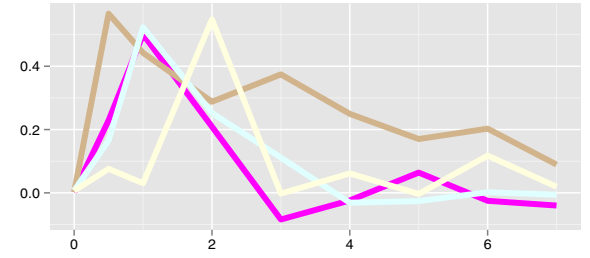

Temporal Cluster

magenta_1hrUp (Area X, HVC)

- tan_transientEarlyUp (All)

lightcyan_1to2hrlncrease (All)

lightyellow_2hrlncrease (All)

B

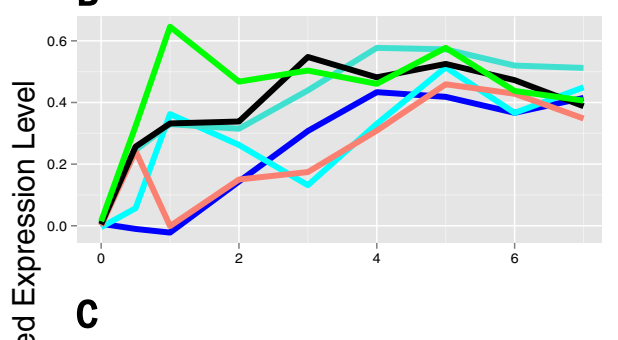

Temporal Cluster

- blue_delayedUp (Area X, HVC)

- cyan_lateDoublelncrease (HVC)

- turquoise_slowUp (Area X)

- salmon_doublelncrease (RA)

- black_slowUp (Area X)

- green_sustainedUp (Area X)

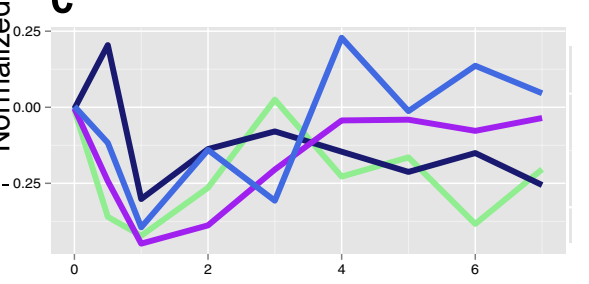

Temporal Cluster

_- lightgreen_inconsistentDecrease (RA)

midnightblue_inconsistentDecrease (Area X, HVC, RA)

purple_transientDecrease (Area X, HVC)

- royalblue_earlyIncreaseLatelncrease (LMAN)

\section{D}

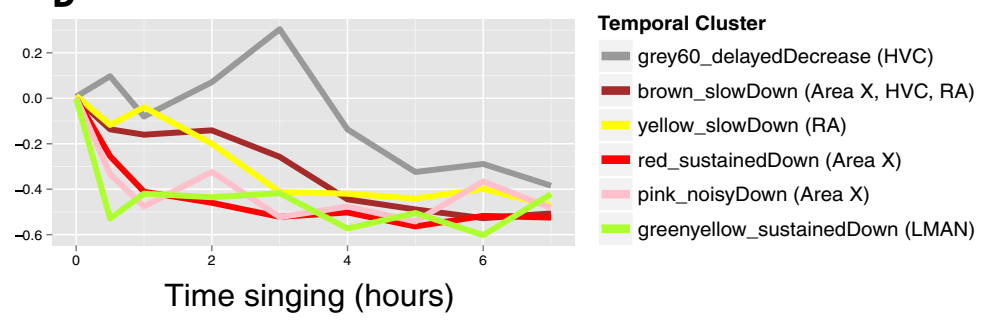

Fig. 5. Temporal singing-regulated patterns across time. (A) Averages of gene expression levels in four temporal clusters of transient early response increases. (B) Averages of six late-response gene cluster increases. (C) Averages of four transient early-response cluster decreases. (D) Averages of six late-response gene cluster decreases. The temporal profiles are normalized such that nonsinging birds have a value of 0 and each gene has a maximum increase or decrease of 1 . Each point represents the mean across all gene-brain region combinations for that time point. The 20 colors match the major temporal clusters in fig. S3, A to D.

begins are predictive of differential singingdependent induction of late-response genes. This hypothesis was further supported by our observation of regional $\mathrm{H} 3 \mathrm{~K} 27 \mathrm{ac}$ differences at baseline for 50 genes that had equivalent basal expression in RA and Area X but region-specific up-regulation upon singing (table S22, blue and red highlights). An ingenuity pathway analysis on the Area X set of genes out of the 50 mentioned above (table S22, blue, and SM15) revealed that they were enriched for locomotion behavior $(P=0.004$; ARNTL, CALB1, FGF14, RCAN2, and RIMS1) and movement-disorder functions $(P=0.004 ; A R N T L, C A L B 1, C A P Z B, D I R A S 2$, EEF1A2, ELMO1, FGF14, MTMR2, RPSA, and $T M E D 10)$, consistent with the function of Area $\mathrm{X}$ and the surrounding striatum. There were too few RA-specific genes without baseline differential expression (10 genes) to be tested by pathway analyses. Overall, these findings indi- that interactions between early transcription factors and late-response genes coordinate activitydependent gene induction associated with behavior (57) but, in this case, in a regionspecific manner. The second, epigenetic, mechanism is just beginning to be explored at the level of neural activity $(40,58)$ and has not been addressed in complex behaviors.

Given our findings and known signaling pathways from experiments in cultured cells (59), we propose the following overall mechanism (see the figure in the print summary, page 1334). Neural activity during the performance of a behavior, such as singing, causes release of neurotransmitters at the synapses between connected cells and activates postsynaptic receptors. These receptors initiate an intracellular signaling response that alters the activity, often through phosphorylation, of constitutively expressed EATFs. The activated EATFs bind or are already bound to the open chromatin of promoters or enhancers of the core IEGs enabled in all brain regions, as measured by H3K27ac, to activate their expression. The IEGs in turn, along with EATFs, bind to recognition regions of open chromatin that have already been primed in a cell type-specific manner, which leads to the induction of regionspecific late-response genes. Some transcription factors are already expressed in a region-specific manner and add to the diversity of regulation of the downstream genes. Furthermore, our data show that brain region-specific open enhancers or promoters are already waiting in an active state, ready to do their job at a moment's notice when the neurons fire to turn on programs of gene expression. Thus, the production of learned behavior modulates an already primed transcriptional and epigenetic network specific to different subregions of the circuit that controls the behavior.

This model may be an explanation for the finding that the IEG and EATF NPAS4, in response to neural activity, activates different sets of genes in cultured excitatory versus inhibitory neurons (60). Likewise, we find that common induction of IEGs across the many different kinds of neurons that comprise all song nuclei is associated with distinct programs of late-response genes, which are likely dependent at least in part on IEG regulation. However, one notable difference between our data and a recent study of activity-dependent enhancers in cultured neuron preparations is that, whereas membrane depolarization was found to further induce $\mathrm{H} 3 \mathrm{~K} 27 \mathrm{ac}$ at enhancers near activity-regulated genes (58), we find that H3K27ac peaks in vivo in the brain are already enriched near singing-inducible genes under basal conditions and do not show further activation upon singing. It is possible that the neural networks recruited upon singing are sparse enough in the song nuclei that we were unable to detect H3K27ac changes in these cells against the background noise. An alternative possibility is that ongoing neural activity in the brain of an awake behaving animal is sufficient to keep enhancers poised in a fully active state even before execution of a specific behavioral task like 

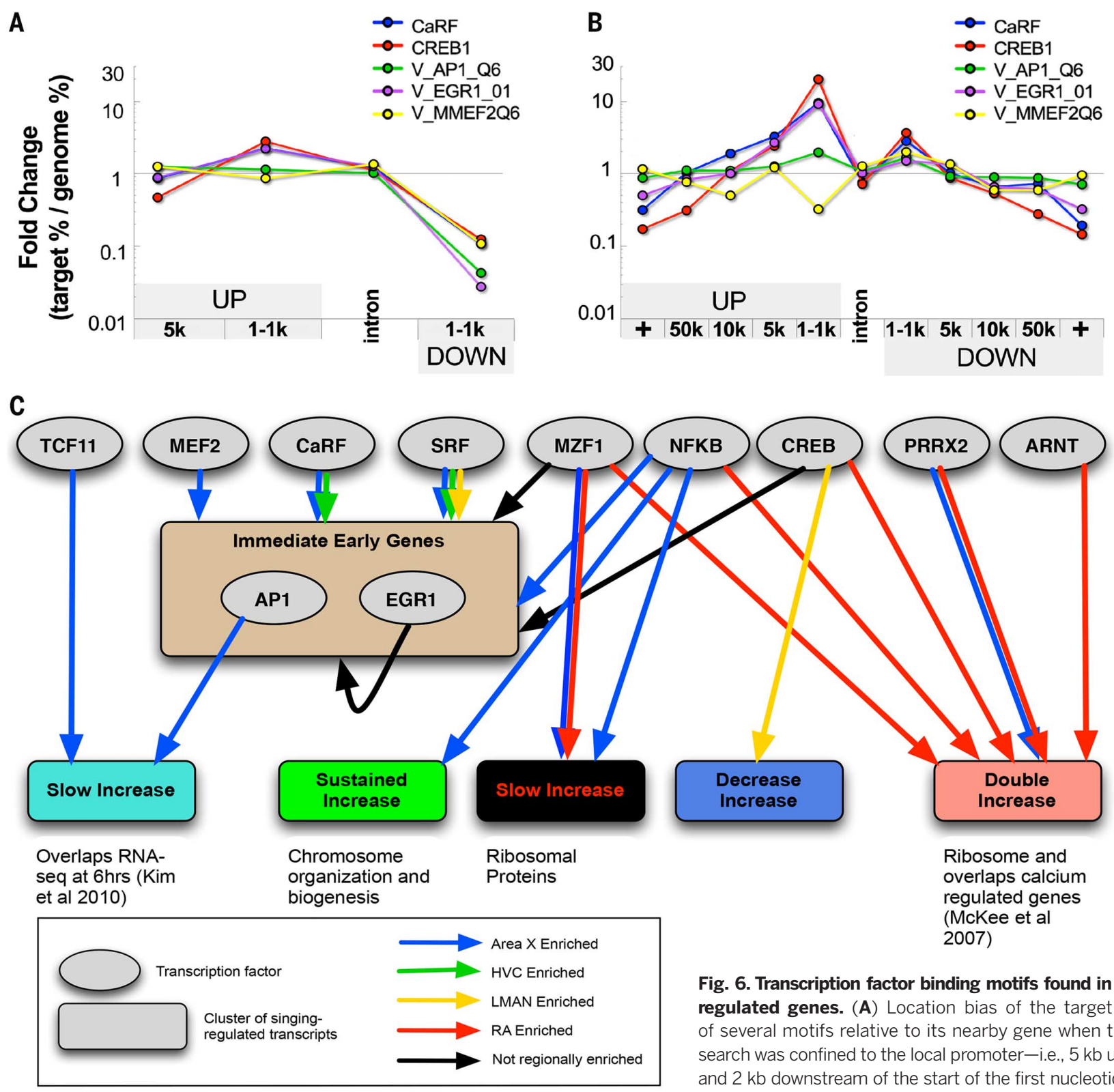
DOWN

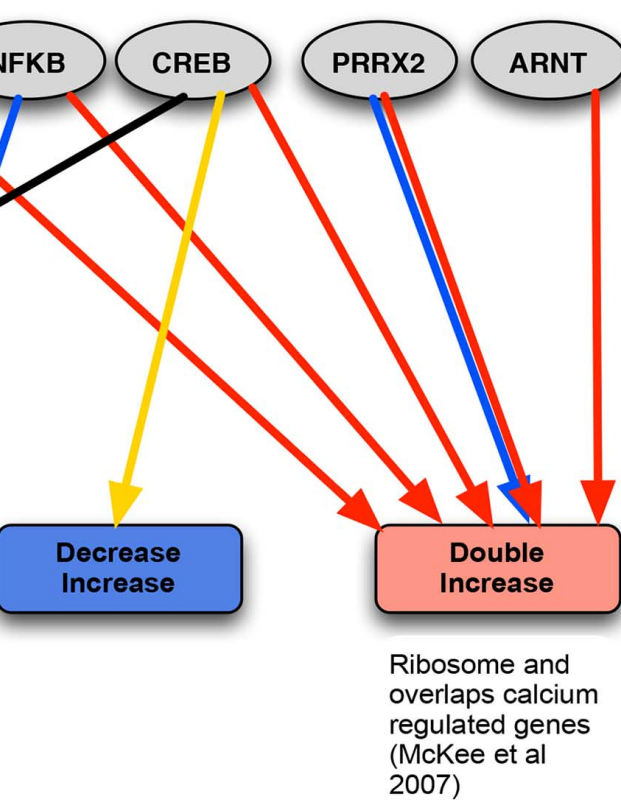

Fig. 6. Transcription factor binding motifs found in singingregulated genes. (A) Location bias of the target window of several motifs relative to its nearby gene when the motif search was confined to the local promoter-i.e., $5 \mathrm{~kb}$ upstream and $2 \mathrm{~kb}$ downstream of the start of the first nucleotide of the first exon of the gene. Fold change (plotted on the log scale $y$ axis) is the ratio of the percentage of the motif target windows that fell within a particular position category relative to the first exon of a gene (target \%) versus the percentage of windows that fall within that position category genome-wide (genome \%). (B) Location bias of the motif target window relative to its nearby gene when the motif search was performed over the gene territory-i.e., halfway upstream and halfway downstream to the last or first exon of the nearest nonoverlapping gene. (C) Transcription factor motif-gene cluster network summarized from fig. S4 showing relationships between enriched EATFs (gray circles) and their binding motifs in subsets of genes from the temporal singing-regulated clusters (colored rectangular nodes as in fig. S3, A to D). Edges are colored on the basis of the region-specific expression of the predicted regulatory targets of the TF within each singing-regulated cluster (SM11 and SM12). Detailed results are in table S13 and fig. S4.

singing. In this model, it is regulation of sequencespecific DNA binding of transcription factors that is most important for instructing the level and nature of gene expression, whereas epigenetic marks on chromatin are permissive for expression of the predetermined program.

Our CaRF manipulation experiments help reveal further complexity and potential novel mechanisms of activity-dependent gene regulation in the brain. The increased activity-regulated genes that are reversed in the absence of $\mathrm{CaRF}$ in response to membrane depolarization sug- gest that $C a R F$ may act as a modulating transcription factor for neural activity-dependent regulation of its target genes. In this scenario, it prevents differential expression of its target genes until neural firing increases. When $C a R F$ is removed by knockdown, it can no longer buffer the expression of these genes in the absence of activity; consequently, in the presence of activity, other factors can regulate the genes in a direction opposite of what $C a R F$ would do. The specific mechanisms by which $C a R F$ might achieve this function remain to be determined, but the H3K27ac enhancer activity in $\mathrm{CaRF}$ target genes is likely to play a role.

Additional transcriptional anatomical diversity not tested in this study could possibly be generated with differential expression of neurotransmitter membrane receptors at baseline in different brain regions, which could activate different signaling pathways in those neurons during singing $(2,33)$. Our hypothesis does not explain the down-regulation of some gene clusters where regionally specific transcription factor motifs were not enriched in those genes, and 
A

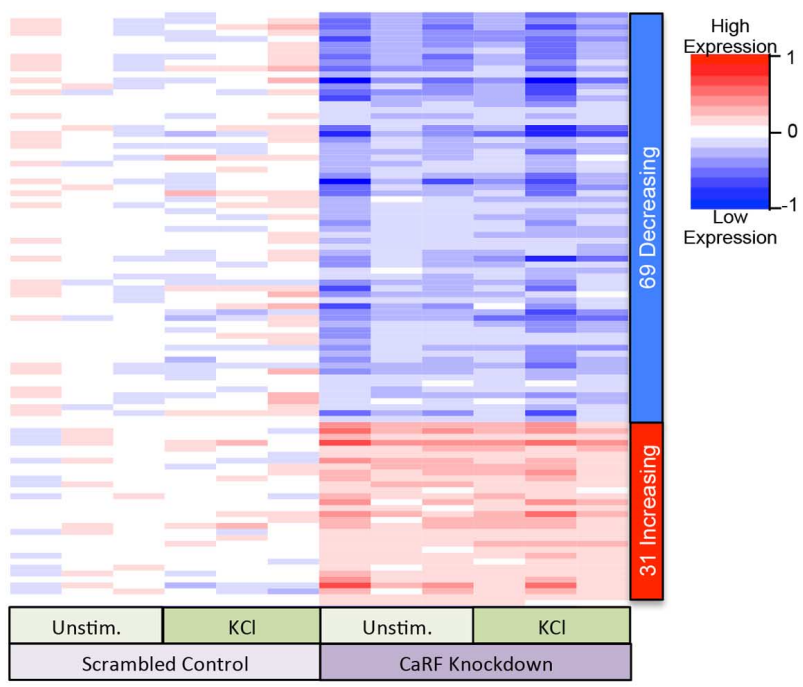

C

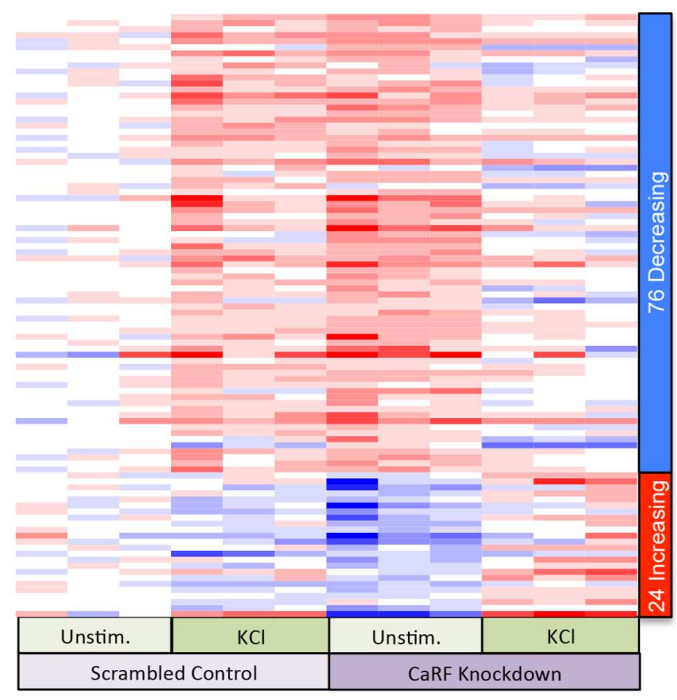

Fig. 7. RNAi knockdown illuminates CaRF binding motif relationships with singing-regulated genes. (A) Heat map of genes affected by CaRF knockdown independent of membrane depolarization in mouse cultured neurons. Rows represent the 100 transcripts most changed by CaRF RNAi knockdown $(P<$ 0.0014; FDR $q<0.475)$, sorted according to the $t$ statistic, which takes direction of regulation into account. Each column is an independent sample $(n=3$ unstimulated controls; $n=3 \mathrm{KCl}$ depolarized in the presence of either scrambled RNAi or CaRF RNAi knockdown virus). Color intensities (blue to red) represent the log fold change in knockdown cells relative to the mean of the scrambled control conditions. (B) Significance of the enrichment of zebra finch baseline genes (cluster colors according to Fig. 2A) with CaRF promoter motifs in the
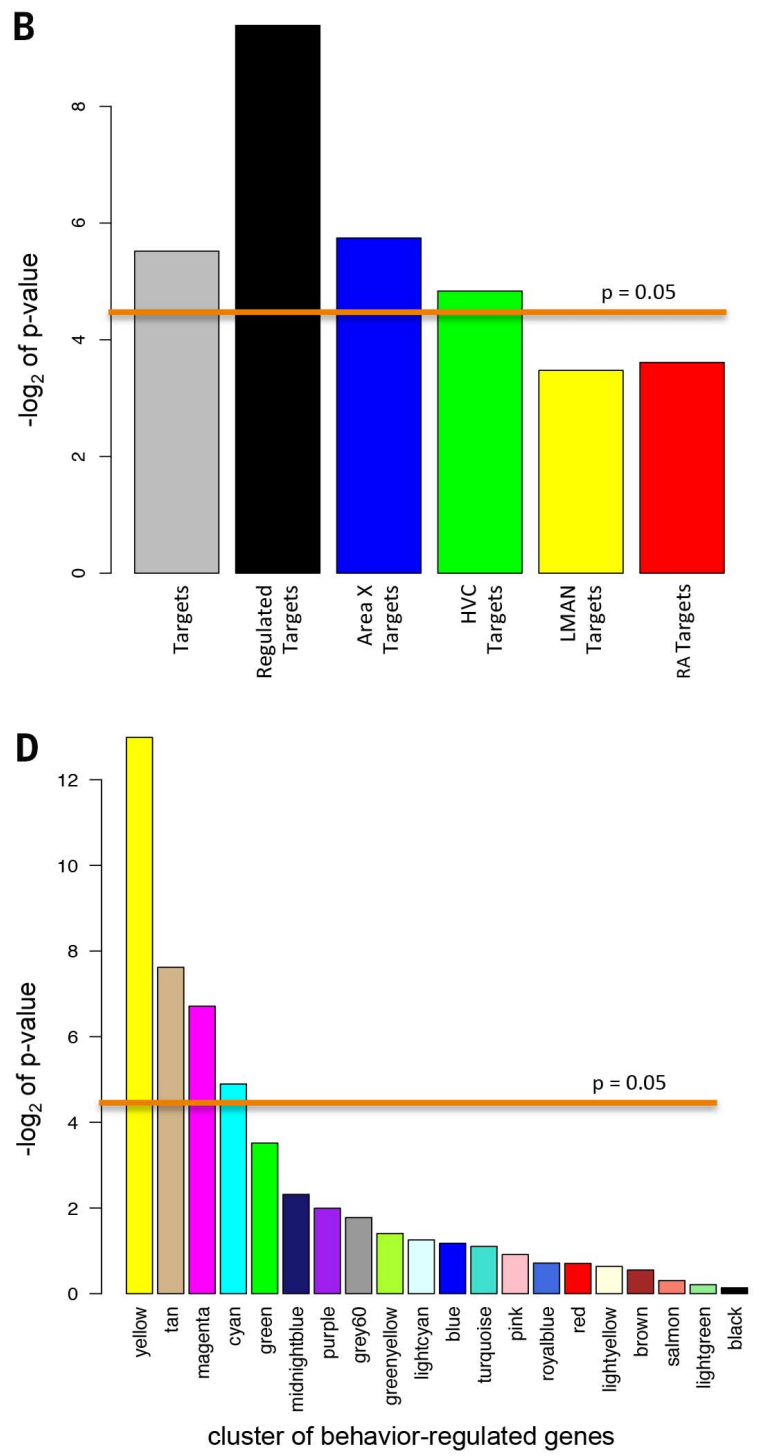

ranked list of $t$ values for CaRF knockdown-affected genes in mouse cultured neurons. $P<0.05$ (above line) is a significant association, Wilcox rank sum statistic over multiple permutations (66). (C) Similar to (A), except for genes that respond differently to $\mathrm{KCl}$ activity in the CaRF knockdown cells. Rows represent the 100 transcripts most changed in expression $(P<0.015$, factorial test), sorted according to the $t$ statistic. (D) Significance of the enrichment of zebra finch singing-regulated genes (cluster colors according to Fig. 5 and fig. S3), with CaRF promoter motifs in the ranked list of $t$ values for genes differentially regulated by neural activity in mouse cortical neurons during CaRF knockdown versus control. $P<0.05$ (above line) is a significant association, Wilcox rank sum statistic over multiple permutations (66). thus their regulation would have to be explained by other mechanisms.

Our findings suggest that each song nucleus has diverse molecular functions and gene networks. Consistent with their dominant roles in song production (7-13) compared to other song nuclei, HVC is specifically enriched with singingregulated increases in PSD proteins used for cell-to-cell communication and RNA-protein complexes, and RA is enriched with genes in the mitogen-activated protein kinase (MAPK) pathway, such as DUSP1, which is proposed to be involved in neural protection of a brain region that is highly active during behavior performance $(61,62)$. Consistent with their dominant roles in learning (7-13), LMAN shows greater specificity for the cAMP response element-binding protein (CREB) pathway, a key transcription factor involved in learning and memory $(59,63)$, and Area $\mathrm{X}$ is more enriched with expression of neural connectivity, chromosome organization, and biogenesis genes. In addition, the large overrepresentation of noncoding RNA genes expressed at baseline in Area X indicates that its transcrip- tional regulatory network may be more extensive than the pallial song nuclei. The larger overrepresentation of neural connectivity and cell signaling genes in the pallial song nuclei indicates greater focus on cell structure and communication.

In terms of memory, a long-held hypothesis is that neural activity will induce an early wave of responsive genes, which in turn regulate a late wave of genes, and that the first wave would act as a molecular switch converting short-term memories into long-term memories $(57,64,65)$. 
If true, singing would be associated with continuous memory consolidation and song finetuning, with each nucleus having specific waves of gene regulation for their specific functions.
An alternative, not mutually exclusive, proposal states that the activity-dependent waves function as a metabolic mechanism to maintain protein turnover for normal cell homeostasis due to increased protein catabolism that occurs during high activity levels (17). If true, it would be associated with continued repair of the circuit when used. Our transcription factor binding motif

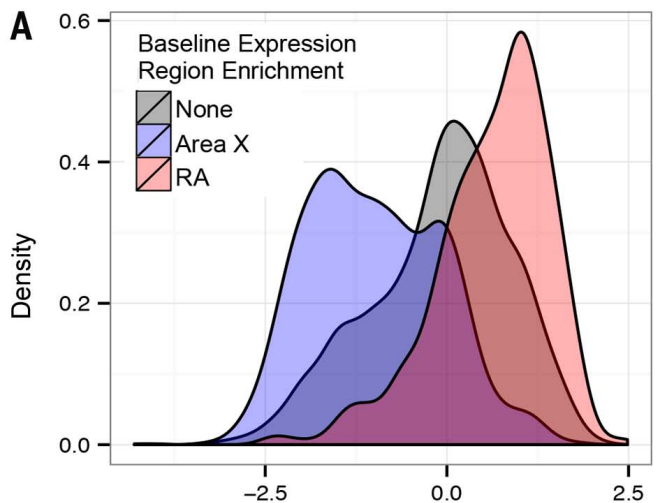

Mean Log2 H3K27ac peak signal in RA vs. Area X

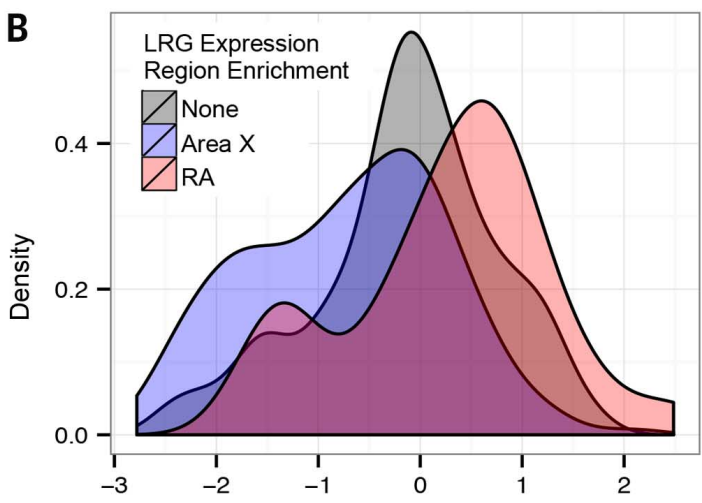

Mean Log2 H3K27ac peak signal in RA vs. Area X

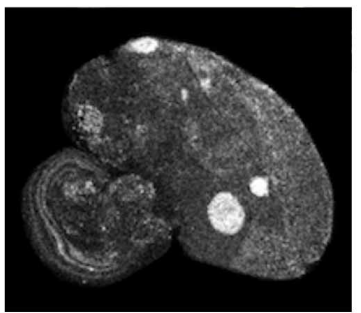

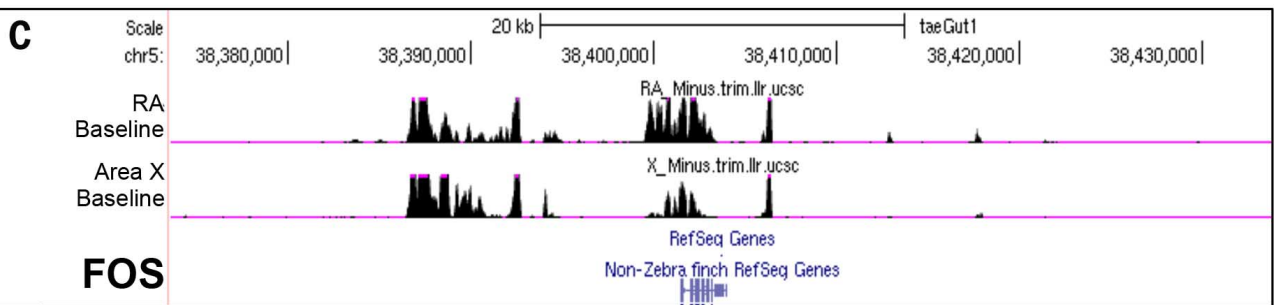
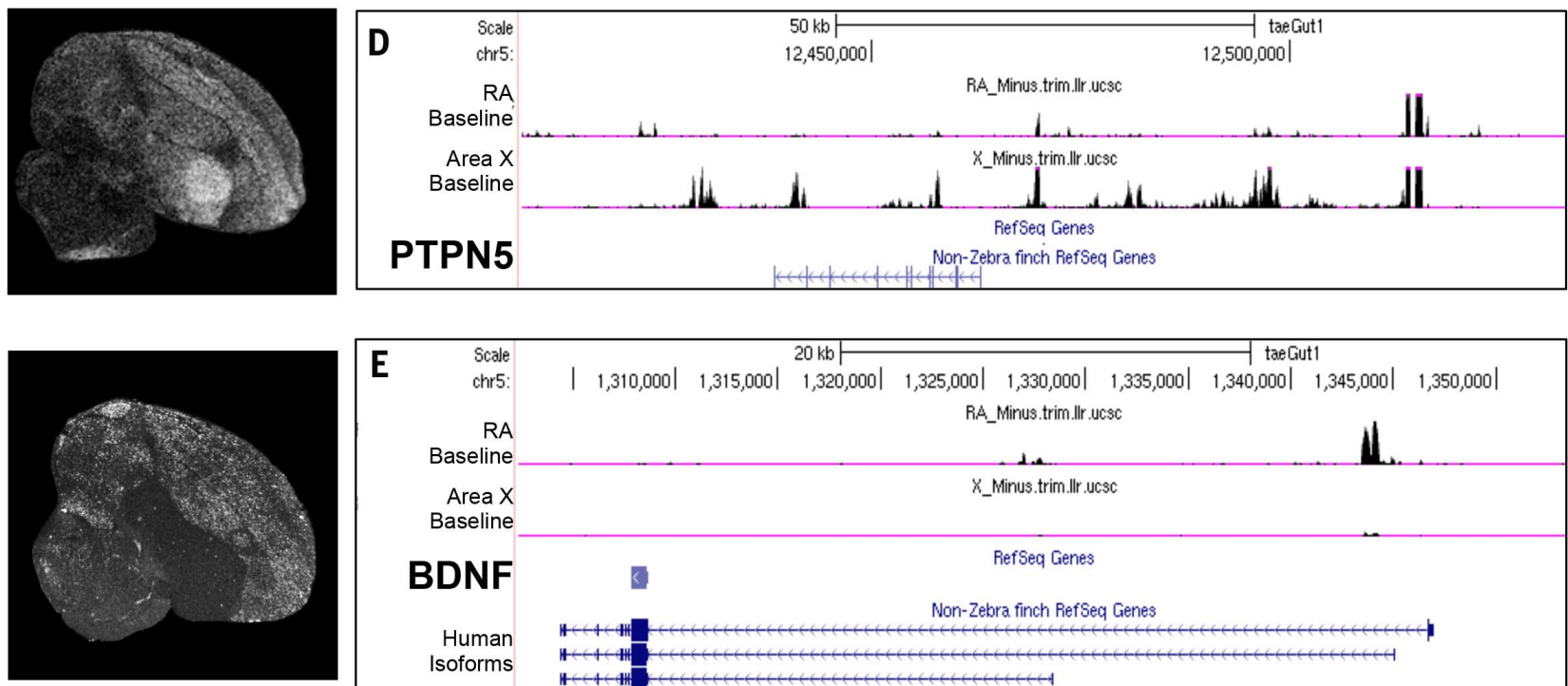

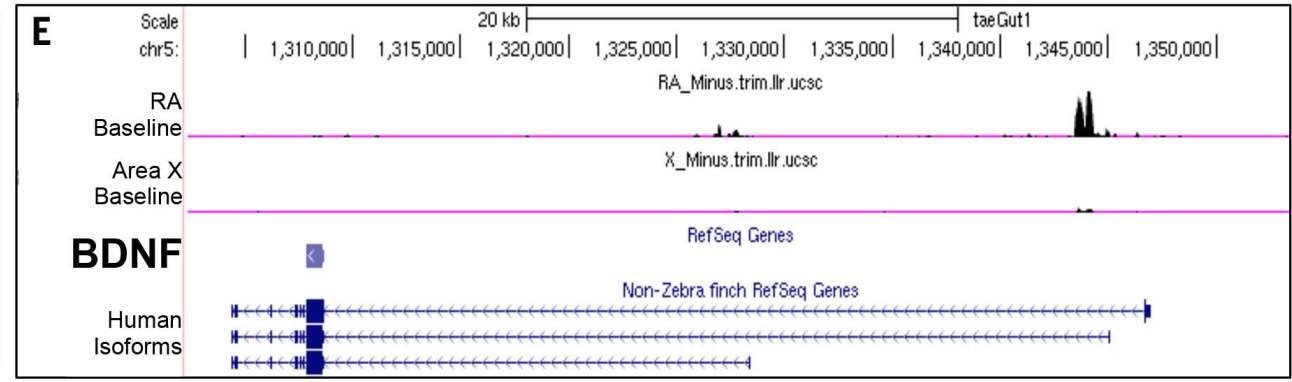

Fig. 8. Region-specific epigenetic signatures predefine behaviorally regulated gene expression. (A) Density plot of genes differentially expressed at baseline in RA versus Area $X$ and the difference in the level of nearby H3K27ac peaks in the genomes of cells in RA versus Area X. Each H3K27ac peak is mapped to a gene with the nearest transcription start site. For each gene, the changes in all mapped H3K27ac peaks are averaged. The H3K27ac distributions for $R A$ versus Area $X$ enriched genes are significantly different $\left(P=1.5 \times 10^{-186}, t\right.$ test). (B) Similar plot as in (A) except for differentially expressed late-response singing-regulated genes. The distributions for RA and Area $X$ are also significantly different $\left(P=1.8 \times 10^{-5}, t\right.$ test). However, there are two peaks in RA, which suggests that active genomic sites in Area $X$ in the negative peak for RA could be genes that are actively suppressed in Area X. Corresponding data can be found in tables S21 and S22. (C) H3K27ac peaks surrounding a gene induced by singing across all brain regions, FOS; (D) H3K27ac peaks of a gene induced specifically in Area X, PTPN5. (E) H3K27ac peaks of a gene induced at low levels in RA but not detectable in Area X, BDNF. The plots show the log-likelihood ratios of H3K27ac signal in pooled baseline RA and pooled baseline Area $X$ samples versus input DNA around the genomic regions in the zebra finch. The relevant gene models from the UCSC genome browser are shown below. Peaks measure both enhancer and promoter regions. Left of the H3K27ac peaks are in situ hybridization mRNA signal in singing animals. FOS and PTPN5 are shown in Fig. 3, and BDNF is used with permission from (37). 
analysis suggests that both the early and late transcriptional responses could be driven by some of the same EATFs. This would indicate that the two waves of gene expression may not entirely depend on each other and that they could be used for both memory and homeostasis functions.

In summary, as the mechanisms that define the genome-phenotype relationship, including the diversity of gene expression patterns, begin to be understood, so will the role of individual genes and pathways in learning, maintenance, and production of behavior. Performance of complex behavior involves interaction between neural activity, networks of cells, and networks of genes. Untangling the subtle differences in connected neurons, firing patterns, signaling pathways, and transcription factor activity may lead to a greater understanding of the diversity of the gene expression patterns that we observe here in highly interconnected cells within an intact multicellular organ.

\section{REFERENCES AND NOTES}

1. A. J. Doupe, P. K. Kuhl, Birdsong and human speech: Common themes and mechanisms. Annu. Rev. Neurosci. 22, 567-631 (1999). doi: 10.1146/annurev.neuro.22.1.567; pmid: 10202549

2. E. D. Jarvis, Learned birdsong and the neurobiology of human language. Ann. N. Y. Acad. Sci. 1016, 749-777 (2004). doi: 10.1196/annals.1298.038; pmid: 15313804

3. R. Mooney, M. Konishi, Two distinct inputs to an avian song nucleus activate different glutamate receptor subtypes on individual neurons. Proc. Natl. Acad. Sci. U.S.A. 88, 4075-4079 (1991). doi: 10.1073/pnas.88.10.4075; pmid: 11607180

4. M. Luo, D. J. Perkel, A GABAergic, strongly inhibitory projection to a thalamic nucleus in the zebra finch song system. J. Neurosci. 19, 6700-6711 (1999). pmid: 10414999

5. A. Reiner et al., Revised nomenclature for avian telencephalon and some related brainstem nuclei. J. Comp. Neurol. 473, 377-414 (2004). doi: 10.1002/cne.20118 pmid: 15116397

6. E. D. Jarvis et al., Avian brains and a new understanding of vertebrate brain evolution. Nat. Rev. Neurosci. 6, 151-159 (2005). doi: 10.1038/nrn1606; pmid: 15685220

7. F. Nottebohm, T. M. Stokes, C. M. Leonard, Central control of song in the canary, Serinus canarius. J. Comp. Neurol. 165, 457-486 (1976). doi: 10.1002/cne.901650405; pmid: 1262540

8. S. W. Bottjer, E. A. Miesner, A. P. Arnold, Forebrain lesions disrupt development but not maintenance of song in passerine birds. Science 224, 901-903 (1984). doi: 10.1126/ science.6719123; pmid: 6719123

9. C. Scharff, F. Nottebohm, A comparative study of the behavioral deficits following lesions of various parts of the zebra finch song system: Implications for vocal learning. J. Neurosci. 11, 2896-2913 (1991). pmid: 1880555

10. H. Williams, N. Mehta, Changes in adult zebra finch song require a forebrain nucleus that is not necessary for song production. J. Neurobiol. 39, 14-28 (1999). doi: 10.1002/ (SICI)1097-4695(199904)39:1<14::AID-NEU2>3.0.CO;2-X; pmid: 10213450

11. M. S. Brainard, A. J. Doupe, Interruption of a basal ganglia-forebrain circuit prevents plasticity of learned vocalizations. Nature 404, 762-766 (2000). doi: 10.1038/ 35008083: pmid: 10783889

12. B. P. Olveczky, A. S. Andalman, M. S. Fee, Vocal experimentation in the juvenile songbird requires a basal ganglia circuit. PLOS Biol. 3, e153 (2005). doi: 10.1371 journal.pbio.0030153; pmid: 15826219

13. D. Aronov, A. S. Andalman, M. S. Fee, A specialized forebrain circuit for vocal babbling in the juvenile songbird. Science 320, 630-634 (2008). doi: 10.1126/science.1155140; pmid: 18451295

14. S. W. Bottjer, F. Johnson, Circuits, hormones, and learning: Vocal behavior in songbirds. J. Neurobiol. 33, 602-618 (1997). doi: 10.1002/(SICI)1097-4695(19971105)33:5<602:: AID-NEU8>3.0.C0/2-8; pmid: 9369462
15. A. R. Pfenning et al., Convergent transcriptional specializations in the brains of humans and song learning birds. Science 346, 1256846 (2014).

16. C. Scharff, J. Petri, Evo-devo, deep homology and FoxP2 Implications for the evolution of speech and language. Philos. Trans. R. Soc. Lond. B Biol. Sci. 366, 2124-2140 (2011). doi: 10.1098/rstb.2011.0001; pmid: 21690130

17. E. D. Jarvis, F. Nottebohm, Motor-driven gene expression. Proc. Natl. Acad. Sci. U.S.A. 94, 4097-4102 (1997) doi: 10.1073/pnas.94.8.4097; pmid: 9108111

18. R. R. Kimpo, A. J. Doupe, FOS is induced by singing in distinct neuronal populations in a motor network. Neuron 18 315-325 (1997). doi: 10.1016/S0896-6273(00)80271-8; pmid: 9052801

19. H. Jin, D. F. Clayton, Localized changes in immediate-early gene regulation during sensory and motor learning in zebra finches. Neuron 19, 1049-1059 (1997). doi: 10.1016/ S0896-6273(00)80396-7; pmid: 9390518

20. E. D. Jarvis, C. Scharff, M. R. Grossman, J. A. Ramos, F. Nottebohm, For whom the bird sings: Context-dependent gene expression. Neuron 21, 775-788 (1998). doi: 10.1016/ S0896-6273(00)80594-2; pmid: 9808464

21. O. Whitney, K. Soderstrom, F. Johnson, Post-transcriptional regulation of zenk expression associated with zebra finch vocal development. Brain Res. Mol. Brain Res. 80, 279-290 (2000). doi: 10.1016/S0169-328X(00)00178-9; pmid: 11038263

22. 0 . Whitney, F. Johnson, Motor-induced transcription but sensory-regulated translation of ZENK in socially interactive songbirds. J. Neurobiol. 65, 251-259 (2005). doi: 10.1002/ neu.20187; pmid: 16155900

23. L. Kubikova, E. A. Turner, E. D. Jarvis, The pallial basal ganglia pathway modulates the behaviorally driven gene expression of the motor pathway. Eur. J. Neurosci. 25. 2145-2160 (2007). doi: 10.1111/j.1460-9568.2007.05368.x: pmid: 17419760

24. K. Wada et al., A molecular neuroethological approach for identifying and characterizing a cascade of behaviorally regulated genes. Proc. Natl. Acad. Sci. U.S.A. 103 15212-15217 (2006). doi: 10.1073/pnas.0607098103; pmid: 17018643

25. X. C. Li, E. D. Jarvis, B. Alvarez-Borda, D. A. Lim F. Nottebohm, A relationship between behavior, neurotrophin expression, and new neuron survival. Proc. Natl. Acad. Sci. U.S.A. 97, 8584-8589 (2000). doi: 10.1073/ pnas.140222497; pmid: 10890902

26. A. J. Lombardino, X.-C. Li, M. Hertel, F. Nottebohm, Replaceable neurons and neurodegenerative disease share depressed UCHL1 levels. Proc. Natl. Acad. Sci. U.S.A. 102, 8036-8041 (2005). doi: 10.1073/pnas.0503239102; pmid: 15911766

27. I. Teramitsu, S. A. White, FoxP2 regulation during undirected singing in adult songbirds. J. Neurosci. 26 , 7390-7394 (2006). doi: 10.1523/JNEUROSCI.1662-06.2006; pmid: 16837586

28. J. E. Miller et al., Birdsong decreases protein levels of FoxP2, a molecule required for human speech. J. Neurophysiol. 100, 2015-2025 (2008). doi: 10.1152/jn.90415.2008; pmid: 18701760

29. W. C. Warren et al., The genome of a songbird. Nature 464, 757-762 (2010). doi: 10.1038/nature08819: pmid: 20360741

30. A. T. Hilliard, J. E. Miller, E. R. Fraley, S. Horvath, S. A. White, Molecular microcircuitry underlies functional specification in a basal ganglia circuit dedicated to vocal learning. Neuron 73, 537-552 (2012). doi: 10.1016/ j.neuron.2012.01.005; pmid: 22325205

31. G. Zhang et al., Comparative genomics reveals insights into avian genome evolution and adaptation. Science 346, 1311-1320 (2014)

32. S. Haesler et al., FoxP2 expression in avian vocal learners and non-learners. J. Neurosci. 24, 3164-3175 (2004). doi: 10.1523/JNEUROSCI.4369-03.2004; pmid: 15056696

33. K. Wada, H. Sakaguchi, E. D. Jarvis, M. Hagiwara, Differential expression of glutamate receptors in avian neural pathways for learned vocalization. J. Comp. Neurol. 476 44-64 (2004). doi: 10.1002/cne.20201; pmid: 15236466

34. L. Kubikova, K. Wada, E. D. Jarvis, Dopamine receptors in a songbird brain. J. Comp. Neurol. 518, 741-769 (2010). doi: 10.1002/cne.22255; pmid: 20058221

35. X. Li et al., Genomic resources for songbird research and their use in characterizing gene expression during brain development. Proc. Natl. Acad. Sci. U.S.A. 104, 6834-6839 (2007). doi: 10.1073/pnas.0701619104; pmid: 17426146
36. P. V. Lovell, D. F. Clayton, K. L. Replogle, C. V. Mello, Birdsong "transcriptomics": Neurochemical specializations of the oscine song system. PLOS ONE 3, e3440 (2008). doi: 10.1371/journal.pone.0003440; pmid: 18941504

37. E. D. Jarvis et al., Global view of the functional molecular organization of the avian cerebrum: Mirror images and functional columns. J. Comp. Neurol. 521, 3614-3665 (2013). doi: 10.1002/cne.23404; pmid: 23818122

38. M. R. Lyons, A. E. West, Mechanisms of specificity in neuronal activity-regulated gene transcription. Prog. Neurobiol. 94, 259-295 (2011). doi: 10.1016/ j.pneurobio.2011.05.003; pmid: 21620929

39. S. W. Flavell et al., Genome-wide analysis of MEF2 transcriptional program reveals synaptic target genes and neuronal activity-dependent polyadenylation site selection. Neuron 60, 1022-1038 (2008). doi: 10.1016/ j.neuron.2008.11.029; pmid: 19109909

40. T.-K. Kim et al., Widespread transcription at neuronal activity-regulated enhancers. Nature 465, 182-187 (2010) doi: 10.1038/nature09033; pmid: 20393465

41. S. Dong et al., Discrete molecular states in the brain accompany changing responses to a vocal signal. Proc. Natl. Acad. Sci. U.S.A. 106, 11364-11369 (2009). doi: 10.1073/ pnas.0812998106; pmid: 19541599

42. G. Feenders et al., Molecular mapping of movement-associated areas in the avian brain: A motor theory for vocal learning origin PLOS ONE 3, e1768 (2008). doi: 10.1371/journal.pone.0001768: pmid: 18335043

43. C. S. Hill, R. Treisman, Growth factors and gene expression: Fresh insights from arrays. Sci. STKE 1999, PE1 (1999). doi: 10.1126/stke.1999.3.pe1; pmid: 11865182

44. A. Barco, J. M. Alarcon, E. R. Kandel, Expression of constitutively active CREB protein facilitates the late phase of long-term potentiation by enhancing synaptic capture. Cell 108, 689-703 (2002). doi: 10.1016/S0092. 8674(02)00657-8; pmid: 11893339

45. S. Sinha, On counting position weight matrix matches in a sequence, with application to discriminative motif finding. Bioinformatics 22, e454-e463 (2006). doi: 10.1093/ bioinformatics/btl227; pmid: 16873507

46. J. Kim et al., Functional characterization of transcription factor motifs using cross-species comparison across large evolutionary distances. PLOS Comput. Biol. 6, e1000652 (2010). doi: 10.1371/journal.pcbi.1000652; pmid: 20126523

47. A. R. Pfenning, R. Schwartz, A. L. Barth, A comparative genomics approach to identifying the plasticity transcriptome. BMC Neurosci. 8, 20 (2007). doi: 10.1186/ 1471-2202-8-20; pmid: 17355637

48. A. R. Pfenning et al., Genome-wide identification of calcium-response factor (CaRF) binding sites predicts a role in regulation of neuronal signaling pathways. PLOS ONE 5, e10870 (2010). doi: 10.1371/journal.pone.0010870; pmid: 20523734

49. S. W. Flavell, M. E. Greenberg, Signaling mechanisms linking neuronal activity to gene expression and plasticity of the nervous system. Annu. Rev. Neurosci. 31, 563-590 (2008). doi: 10.1146/annurev.neuro.31.060407.125631; pmid: 18558867

50. S. Loebrich, E. Nedivi, The function of activity-regulated genes in the nervous system. Physiol. Rev. 89, 1079-1103 (2009). doi: 10.1152/physrev.00013.2009; pmid: 19789377

51. X. Cao, R. Mahendran, G. R. Guy, Y. H. Tan, Detection and characterization of cellular EGR-1 binding to its recognition site. J. Biol. Chem. 268, 16949-16957 (1993). pmid: 8349585

52. 0. Johnsen et al., Small Maf proteins interact with the human transcription factor TCF11/Nrf1/LCR-F1. Nucleic Acids Res. 24, 4289-4297 (1996). doi: 10.1093/nar/24.21.4289; pmid: 8932385

53. A. Subramanian et al., Gene set enrichment analysis: A knowledge-based approach for interpreting genome-wide expression profiles. Proc. NatI. Acad. Sci. U.S.A. 102, 15545-15550 (2005). doi: 10.1073/pnas.0506580102 pmid: 16199517

54. K. A. McDowell et al., Reduced cortical BDNF expression and aberrant memory in Carf knock-out mice. J. Neurosci. 30 7453-7465 (2010). doi: 10.1523/JNEUROSCI.3997-09.2010; pmid: 20519520

55. ENCODE Project Consortium, An integrated encyclopedia of DNA elements in the human genome. Nature 489, 57-74 (2012). doi: 10.1038/nature11247; pmid: 22955616

56. UCSC Genome Browser; https://genome.ucsc.edu/cgi-bin/ hgTracks?hgS_doOtherUser=submit\&hgS_otherUserName= apfenning\&hgS_otherUserSessionName=taeGut1_basic (2014) 
57. P. Goelet, V. F. Castellucci, S. Schacher, E. R. Kandel, The long and the short of long-term memory-A molecular framework. Nature 322, 419-422 (1986). doi: 10.1038/ 322419a0; pmid: 2874497

58. A. N. Malik et al., Genome-wide identification and characterization of functional neuronal activity-dependent enhancers. Nat. Neurosci. 17, 1330-1339 (2014). doi: 10.1038/nn.3808; pmid: 25195102

59. A. E. West, M. E. Greenberg, Neuronal activity-regulated gene transcription in synapse development and cognitive function. Cold Spring Harb. Perspect. Biol. 3, a005744-a005744 (2011). doi: 10.1101/cshperspect. a005744; pmid: 21555405

60. I. Spiegel et al., Npas 4 regulates excitatory-inhibitory balance within neural circuits through cell-type-specific gene programs. Cell 157, 1216-1229 (2014). doi: 10.1016/ j.cell.2014.03.058; pmid: 24855953

61. H. Horita, K. Wada, M. V. Rivas, E. Hara, E. D. Jarvis, The dusp1 immediate early gene is regulated by natural stimuli predominantly in sensory input neurons. J. Comp. Neurol. 518, 2873-2901 (2010). pmid: 20506480

62. H. Horita et al., Specialized motor-driven dusp1 expression in the song systems of multiple lineages of vocal learning birds. PLOS ONE 7, e42173 (2012). doi: 10.1371/ journal.pone.0042173; pmid: 22876306

63. K. Sakamoto, K. Karelina, K. Obrietan, CREB: A multifaceted regulator of neuronal plasticity and protection. J. Neurochem. 116, 1-9 (2011). doi: 10.1111/j.1471-4159.2010.07080.x; pmid: 21044077

64. J. I. Morgan, T. Curran, Stimulus-transcription coupling in neurons: Role of cellular immediate-early genes. Trends Neurosci. 12, 459-462 (1989). doi: 10.1016/ 0166-2236(89)90096-9; pmid: 2479148

65. C. H. Bailey, D. Bartsch, E. R. Kandel, Toward a molecular definition of long-term memory storage. Proc. Natl. Acad. Sci. U.S.A. 93, 13445-13452 (1996). doi: 10.1073/ pnas.93.24.13445; pmid: 8942955

66. J. M. Wettenhall, G. K. Smyth, limmaGUI: A graphical user interface for linear modeling of microarray data. Bioinformatics 20, 3705-3706 (2004). doi: 10.1093/bioinformatics/bth449; pmid: 15297296

\section{ACKNOWLEDGMENTS}

We thank S. Augustin, J. Coleman, and N. Diaz Nelson for help collecting and processing singing bird samples for microarray analysis; H. Dressman and L.-L. Rowlette at the Duke Institute for Genome Science and Policy for microarray hybridizations; We thank A. Cavanaugh, M. Lawson, and M. Dean for useful comments after careful reading of an earlier version of this manuscript. O.W. was supported by postdoctoral training grants from American Psychological Association fellowship funded by the National Institute of Mental Health grant 5T32MH018882-18 and the National Science Foundation grant 0610337 . This work was supported by grants from the National Institute on Deafness and Other Communication Disorders grant R01DC007218 and the Howard Hughes Medical Institute to E.D.J.. Microarray data have been submitted to the Gene Expression Omnibus (www.ncbi.nlm.nih.gov/geo) under accession no. GSE33365. Annotations are also available in table S1. Raw files of the Chip-seq H3K27ac experiments are available at www.broadinstitute.org/ pfenning/finchWig.

\section{SUPPLEMENTARY MATERIALS}

www.sciencemag.org/content/346/6215/1256780/suppl/DC1 Materials and Methods

Figs. S1 to S12

Tables S1 to S22

References (67-117)

2 June 2014; accepted 11 November 2014

10.1126/science. 1256780 

and the singing genome

Osceola Whitney et al.

Science 346, (2014);

DOI: $10.1126 /$ science. 1256780

This copy is for your personal, non-commercial use only.

If you wish to distribute this article to others, you can order high-quality copies for your colleagues, clients, or customers by clicking here.

Permission to republish or repurpose articles or portions of articles can be obtained by following the guidelines here.

The following resources related to this article are available online at www.sciencemag.org (this information is current as of January 14, 2015 ):

Updated information and services, including high-resolution figures, can be found in the online version of this article at:

http://www.sciencemag.org/content/346/6215/1256780.full.html

Supporting Online Material can be found at:

http://www.sciencemag.org/content/suppl/2014/12/11/346.6215.1256780.DC1.html

A list of selected additional articles on the Science Web sites related to this article can be found at:

http://www.sciencemag.org/content/346/6215/1256780.full.html\#related

This article cites 114 articles, 50 of which can be accessed free:

http://www.sciencemag.org/content/346/6215/1256780.full.html\#ref-list-1

This article has been cited by 2 articles hosted by HighWire Press; see:

http://www.sciencemag.org/content/346/6215/1256780.full.html\#related-urls

This article appears in the following subject collections:

Evolution

http://www.sciencemag.org/cgi/collection/evolution 